\title{
Quadrant Standardization for Herbaceous Species of Benhama, Ganderbal Area in Kashmir
}

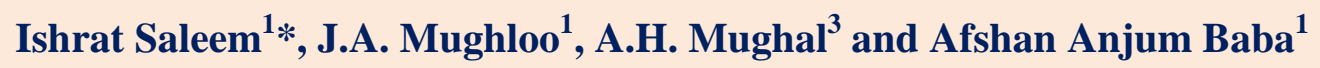 \\ ${ }^{1}$ Faculty of forestry, SKUAST-K India-190025 \\ ${ }^{2}$ Faculty of Agriculture, SKUAST-K India-190025 \\ *Corresponding author
}

\section{A B S T R A C T}

\section{Keywords}

Quadrant, Size, Standardization, Vegetation

Article Info

Accepted:

12 February 2019

Available Online:

10 March 2019
The present study entitled "Quadrant Standardization for herbaceous species of Benhama Ganderbal, Kashmir" was conducted at Faculty of forestry, Sher-e-Kashmir University of Agricultural Sciences and Technology of Kashmir, Benihama, Ganderbal, Jammu and Kashmir during the year 2015-2016 with the aim to assess the best quadrant size, for herbaceous species at this site. Standardization of quadrant sizes was done for studying vegetation of herbaceous species on that degraded site. Five treatments were taken including five sizes of quadrants viz., $0.5 \mathrm{~m} \times 0.5 \mathrm{~m}, 0.75 \mathrm{~m} \times 0.75 \mathrm{~m}, 1 \mathrm{~m} \times 1 \mathrm{~m}, 1.25 \mathrm{~m} \times$ $1.25 \mathrm{~m}$ and $1.5 \mathrm{~m} \times 1.5 \mathrm{~m}$. Among the five quadrant sizes, $(1.5 \mathrm{~m} \times 1.5 \mathrm{~m})$ quadrant size gave highest density, frequency and abundance which was at par with $(1.25 \mathrm{~m} \mathrm{x} 1.25 \mathrm{~m})$ quadrant size and quadrant size $(1.25 \mathrm{~m} \times 1.25 \mathrm{~m})$ was considered to be the best among all the quadrant sizes.

\section{Introduction}

The method of quadrat sampling is among the oldest techniques in ecology and was first introduced by Pound and Clements in 1898 . The term quadrat is strictly defined as a four sided figure. This term usually refers to any sampling unit, whether circular, hexagonal, or even irregular in outline (Dombois and Ellenberg, 1974). The method has two basic requirements: the area is known and the organisms are relatively immobile during the counting period (Krebs, 1999). Plant ecologists have wrestled with these questions since early studies on the optimal size quadrat to characterize vegetation, and to estimate the abundance of an individual plant species (Gleason, 1920; Clapham, 1932). Further work has continued to examine the statistical efficiencies of quadrats of various sizes and shapes. However, few studies have given consideration to the field efficiency and time costs associated with different sampling designs. While expense is an important aspect of any monitoring effort, it is rarely analyzed in the development of monitoring designs (Hines 1984). Given the growing need to efficiently monitor the abundance of rare, threatened and endangered plant species, plant ecologists, nature preserve managers, and 
agency staff the world over are increasingly faced with the problem of designing sampling programs to estimate plant abundance with some desired level of precision, but with resources that allow only very limited time and effort to be invested (Menges and Gordon, 1996; Phillipi et al., 2001).

The quadrat method of studying vegetation has become an integral part of many of the more important ecological investigations. Pound and Clements (1900) reported that although used occasionally throughout the past century for determining the amount of plant material produced for purposes of enumeration, it was organized into a definite system for the study of the structure and development of vegetation.

Clement (1916) has also reported that with the rapid increase in the number of successional and other ecological studies the use of the quadrat and its modifications is becoming as universal as it is fundamental. Similarly Sampson (1914) by the use of this method has worked out a system of deferred grazing for the ranges in the national forests whereby the forage crop is utilized in such a way as to maintain the lands at their highest state of productiveness and at the same time give the greatest possible returns to the stock industry. Optimum plot size and shape may depend upon the distribution of the species measured (Van Dyne et al., 1963). If several species with greatly varying plant sizes, distributions, and densities are to be measured, more than one quadrat size may be required (Smith et al., 1953).

Data obtained from the use of quadrat methods were used to measure spatial pattern (Nosek 1986), to measure cover of vegetation (Molnar and Nosek 1980), and to make quantitative analysis (Aberdeen 1957). Quadrats were reliable samples of the true plant populations (Anderson et al., 1942).

\section{Materials and Methods}

The study was conducted at Faculty campus of Faculty of Forestry located at Benhama, Ganderbal, Jammu and Kashmir spread over 50 ha at an altitude of $1720 \mathrm{~m}-1843 \mathrm{~m}$ above mean sea level. The study site lies on the southern aspect at $34^{\circ}-16^{\prime} \mathrm{N}$ and $74^{0}-46^{\prime} \mathrm{E}$ longitude. The existing land of the study site composed of three types of land problems namely: degraded under utilized (scrub dominated), degraded pastures/grazing lands, barren rocky/stony wasteland. The study site falls in a mid to high altitude characterized by hot summers and very cold winters. The soil formation and soil deposits are essential prerequisites for the growth and nature of plant life. The effect of climate, topography, parent rock material and time are important in soil formation and soil texture. The soil of the study site is sandy loam in texture; high in organic carbon with slightly neutral in $\mathrm{pH}$ and normal in electrical conductivity (Tahir, 2015)

\section{Sampling procedure}

Sampling was carried out by stratified random sampling. At all the three altitudes selected, five quadrats of size $0.50 \mathrm{~m} \times 0.50 \mathrm{~m}, 0.75 \mathrm{~m}$ $\times 0.75 \mathrm{~m}, 1 \mathrm{~m} \times 1 \mathrm{~m}, 1.25 \mathrm{~m} \times 1.25 \mathrm{~m}$ and 1.5 $\mathrm{m} \times 1.5 \mathrm{~m}$ were laid at three places and were replicated thrice, thus making a total of 135 quadrats at the selected site

Following observations were recorded:

\section{Species diversity}

Total number of species present at each quadrant size

\section{Density}

Density number of individuals of a species that occurs within a given sample unit or study area. It was recorded as: 
Number of individuals of

Density $=\frac{\text { the species }}{\begin{array}{l}\text { Total number of quadrats } \\ \text { studied }\end{array}}$

\section{Frequency}

Frequency is the number of times a plant species is present in a given number of sample units. It was calculated by the formula:

\begin{tabular}{|c|c|}
\hline & $\begin{array}{l}\text { Number of quadrats in } \\
\text { which the species } \\
\text { occurred }\end{array}$ \\
\hline & $\begin{array}{l}\text { Total number } \\
\text { quadrats studied }\end{array}$ \\
\hline
\end{tabular}

\section{Abundance}

Abundance is a component of biodiversity and refers to how common or rare a species is relative to other species in a defined location or community. It was calculated by the formula:

\begin{tabular}{ll} 
Abundance & $\begin{array}{l}\text { Total number of } \\
\text { individuals of a species in } \\
\text { all quadrats }\end{array}$ \\
\cline { 2 - 2 }$=$ & $\begin{array}{l}\text { Number of quadrats in } \\
\text { which the species occurred }\end{array}$
\end{tabular}

\section{Results and Discussion}

The data tabulated in the Table 1-4 gives the preliminary information regarding the number of species, their density, frequency and abundance in the study area. The data reveals that treatments had significant effect on the density of herbaceous species. It is evident from the Table 2 that density of species increased with increase in size of quadrant from $\{(0.5 \mathrm{~m} \times 0.5 \mathrm{~m})$ to $(1.5 \mathrm{~m} \times 1.5 \mathrm{~m})\}$. However highest density $\left(607.26 / \mathrm{m}^{2}\right)$ was recorded in $(1.5 \mathrm{~m} \times 1.5 \mathrm{~m})$ quadrant size which was at par with the density $\left(571.52 / \mathrm{m}^{2}\right)$ of species recorded under quadrant size $(1.25 \mathrm{~m}$ $\mathrm{x} 1.25 \mathrm{~m})$ and lowest density $\left(234.67 / \mathrm{m}^{2}\right)$ was recorded under quadrant size $(0.5 \mathrm{~m} \times 0.5 \mathrm{~m})$.

Similarly the data pertaining to the frequency of species increased with increase in size of quadrant from $\{(0.5 \mathrm{~m} \times 0.5 \mathrm{~m})$ to $(1.5 \mathrm{~m} \times$ $1.5 \mathrm{~m})\}$. However highest frequency $(50.7 \%)$ was recorded in quadrant size $(1.5 \mathrm{~m} \times 1.5 \mathrm{~m})$ which was at par with the frequency $(45.83 \%)$ of species recorded under $(1.25 \mathrm{~m} \times 1.25 \mathrm{~m})$ quadrant size and lowest frequency $(10.88 \%)$ was recorded under quadrant size $(0.5 \mathrm{~m} \mathrm{x}$ $0.5 \mathrm{~m})$ (Table 3).

Again the data pertaining to the abundance of species (Table 4) increased with increase in size of quadrant from $\{(0.5 \mathrm{~m} \times 0.5 \mathrm{~m})$ to $(1.5 \mathrm{~m}$ $\mathrm{x} 1.5 \mathrm{~m})\}$. However highest abundance $\left(882.81 / \mathrm{m}^{2}\right)$ was recorded in quadrant size $(1.5 \mathrm{~m} \times 1.5 \mathrm{~m})$ which was at par with the abundance $\left(848 / \mathrm{m}^{2}\right)$ of species recorded under quadrant size $(1.25 \mathrm{~m} \times 1.25 \mathrm{~m})$ and lowest abundance $\left(436 / \mathrm{m}^{2}\right)$ was recorded in quadrant size $(0.5 \mathrm{~m} \times 0.5 \mathrm{~m})$.

The data presented in Table 1 to 5 reveals that the vegetative parameters viz: number of species, density, frequency and abundance of the herbaceous species increased with the increase in quadrant size from $0.5 \mathrm{~m} \times 0.5 \mathrm{~m}$ to $1.5 \mathrm{~m} \times 1.5 \mathrm{~m}$ with the highest density, frequency and abundance of species in quadrant size $1.5 \mathrm{~m} \times 1.5 \mathrm{~m}$, which was at par with the density, frequency and abundance of species recorded in the quadrant size $1.25 \mathrm{~m} \mathrm{x}$ $1.25 \mathrm{~m}$. A quadrant delimits an area in which vegetative cover can be estimated, plants counted or species listed. Since plants often grow in clumps, larger quadrants often include more species, thus resulting in maximum density, frequency and abundance of species in them. Another reason behind this may be due to the fact that present study site being a degraded site having sparse vegetative cover, so large quadrants are required for standardization as they include more number of species as compared to smaller quadrants.. 
Table.1 Influence of different sizes of quadrat on species diversity of herbaceous species

\begin{tabular}{|c|c|c|c|c|c|c|}
\hline \multirow{2}{*}{$\begin{array}{c}\text { S. } \\
\text { No. }\end{array}$} & \multirow[t]{2}{*}{ Species } & \multicolumn{5}{|c|}{ Sizes of quadrat } \\
\hline & & $0.5 \mathrm{~m} \times 0.5 \mathrm{~m}$ & $\begin{array}{c}0.75 \mathrm{~m} \times \\
0.75 \mathrm{~m}\end{array}$ & $1 \mathrm{~m} \times 1 \mathrm{~m}$ & $\begin{array}{c}1.25 \mathrm{~m} \times \\
1.25 \mathrm{~m}\end{array}$ & $\begin{array}{c}1.5 \mathrm{~m} \times \\
1.5 \mathrm{~m}\end{array}$ \\
\hline 1 & Achilleamillefolium & - & - & + & + & + \\
\hline 2 & Amaranthusviridis & - & - & - & + & + \\
\hline 3 & Artemisiaindica & - & - & - & + & + \\
\hline 4 & Avenafatua & - & + & + & + & + \\
\hline 5 & Brumusjaponicas & - & + & + & + & + \\
\hline 6 & Capsellabursa pastoris & + & + & + & + & + \\
\hline 7 & Cirsiumarvense & - & - & - & + & + \\
\hline 8 & Centureaiberica & + & + & + & + & + \\
\hline 9 & Chenopodiumalbum & - & - & + & + & + \\
\hline 10 & Convolvulus arvense & + & + & + & + & + \\
\hline 11 & ConyzaCanadensis & + & + & + & + & + \\
\hline 12 & Chaerophyllumvillossum & - & - & - & - & + \\
\hline 13 & Cynodon dactylon & + & + & + & + & + \\
\hline 14 & Erodium cicutarium & - & - & - & + & + \\
\hline 15 & Euphorbia helioscopia & - & - & - & + & + \\
\hline 16 & Gallium aparine & + & + & + & + & + \\
\hline 17 & Geranium pretense & + & + & + & + & + \\
\hline 18 & Hypericum perforatum & - & - & - & + & + \\
\hline 19 & Lactuca dissecta & + & + & + & + & + \\
\hline 20 & Lepidium rudrale & + & + & + & + & + \\
\hline 21 & Lespedizacapitata & + & + & + & + & + \\
\hline 22 & Lolliumspp & - & - & - & + & + \\
\hline 23 & Marubiumvulgare & + & + & + & + & + \\
\hline 24 & Medicago minima & + & + & + & + & + \\
\hline 25 & Medicagopolymorpha & - & + & + & + & + \\
\hline 26 & Oenotherarosea & - & - & + & + & + \\
\hline 27 & Oxalis corniculata & + & + & + & + & + \\
\hline 28 & Plantagolanceolata & + & + & + & + & + \\
\hline 29 & Poabulbusa & + & + & + & + & + \\
\hline 30 & Pоаanпиа & + & + & + & + & + \\
\hline 31 & Polygonumplebeium & + & + & + & + & + \\
\hline 32 & Prunella vulgaris & + & + & + & + & + \\
\hline 33 & Ranunculus arvense & + & + & + & + & + \\
\hline 34 & Rhumexhistatus & - & - & - & + & + \\
\hline 35 & Rumexnepalensis & - & + & + & + & + \\
\hline 36 & Salvia moorcraftiana & - & - & - & + & + \\
\hline 37 & Scandix pectin veneris & - & - & - & + & + \\
\hline 38 & Sonchusoleraceous & - & + & + & + & + \\
\hline 39 & Solanumnigrum & - & - & + & + & + \\
\hline 40 & Sorghum halepensis & - & - & - & - & + \\
\hline 41 & Stellaria media & - & + & + & + & + \\
\hline 42 & Taraxicumofficinale & - & - & + & + & + \\
\hline 43 & Thymus linearis & - & - & + & + & + \\
\hline 44 & Trifoliumpretense & - & + & + & + & + \\
\hline 45 & Trifoliumrepens & + & + & + & + & + \\
\hline 46 & VerbascumThapsus & - & - & + & + & + \\
\hline 47 & Veronica persica & - & - & - & + & + \\
\hline 48 & Xanthium spinosum & - & - & - & - & + \\
\hline & Total & 20 & 27 & 34 & 45 & 48 \\
\hline
\end{tabular}


Table.2 Influence of different sizes of quadrat on density (plants $/ \mathrm{m}^{2}$ ) of herbaceous species

\begin{tabular}{|c|c|c|c|c|c|c|}
\hline \multirow{2}{*}{$\begin{array}{l}\text { S. } \\
\text { No. }\end{array}$} & \multirow[t]{2}{*}{ Species } & \multicolumn{5}{|c|}{ Sizes of quadrat } \\
\hline & & $\underset{\mathrm{m}}{0.5 \mathrm{~m} \times 0.5}$ & $\begin{array}{c}0.75 \mathrm{~m} \times \\
0.75 \mathrm{~m}\end{array}$ & $1 \mathrm{~m} \times 1 \mathrm{~m}$ & $\begin{array}{c}1.25 \mathrm{~m} \times \\
1.25 \mathrm{~m}\end{array}$ & $\begin{array}{r}1.5 \mathrm{~m} \times \\
1.5 \mathrm{~m}\end{array}$ \\
\hline $\mathbf{1}$ & Achilleamillefolium & - & - & 1.44 & 1.63 & 1.67 \\
\hline 2 & Amaranthusviridis & - & - & - & 8.96 & 9.03 \\
\hline 3 & Artemisia indica & - & - & - & 3.41 & 3.70 \\
\hline 4 & Avenafatua & - & 3.56 & 4.67 & 9.81 & 8.59 \\
\hline 5 & Brumusjaponicas & - & 4.74 & 10 & 9.38 & 8.89 \\
\hline 6 & Capsella bursa pastoris & 4 & 5.92 & 9 & 8.96 & 6.96 \\
\hline 7 & Cirsiumarvense & - & - & - & 2.77 & 2.51 \\
\hline 8 & Centureaiberica & 5.33 & 9.48 & 6.33 & 7.46 & 7.26 \\
\hline 9 & Chenopodium album & - & - & 2.67 & 2.13 & 2.22 \\
\hline 10 & Convolvulus arvense & 8 & 9.48 & 12.33 & 13.65 & 13.48 \\
\hline 11 & ConyzaCanadensis & 12 & 16.59 & 17 & 20.69 & 21.03 \\
\hline 12 & Chaerophyllumvillossum & - & - & - & - & 6.67 \\
\hline 13 & Cynodondactylon & 125.33 & 129.18 & 129.67 & 164.90 & 206.37 \\
\hline 14 & Erodiumcicutarium & - & - & - & 6.82 & 5.62 \\
\hline 15 & Euphorbia helioscopia & - & - & - & 6.82 & 6.07 \\
\hline 16 & Gallium aparine & 10.67 & 4.74 & 7 & 7.68 & 6.22 \\
\hline 17 & Geranium pretense & 6.67 & 4.74 & 5.67 & 5.97 & 5.92 \\
\hline 18 & Hypericumperforatum & - & - & - & 3.84 & 3.11 \\
\hline 19 & Lactucadissecta & 4 & 4.74 & 5.33 & 6.18 & 6.37 \\
\hline 20 & Lepidiumrudrale & 1.33 & 5.92 & 7.33 & 8.96 & 7.85 \\
\hline 21 & Lespedizacapitata & 1.33 & 5.33 & 7 & 7.25 & 5.48 \\
\hline 22 & Lolliumspp & - & - & - & 7.68 & 8.74 \\
\hline 23 & Marubiumvulgare & 2.67 & 4.14 & 9.67 & 10.67 & 10.67 \\
\hline 24 & Medicago minima & 2. 67 & 9.48 & 10.67 & 17.92 & 15.25 \\
\hline 25 & Medicagopolymorpha & - & 3.556 & 3.33 & 4.69 & 5.03 \\
\hline 26 & Oenotherarosea & - & - & 7.33 & 6.4 & 6.51 \\
\hline 27 & Oxalis corniculata & 2.67 & 4.74 & 8 & 11.09 & 10.37 \\
\hline 28 & Plantagolanceolata & 2. 67 & 7.70 & 7.33 & 11.09 & 8.59 \\
\hline 29 & Poabulbusa & 6.67 & 4.74 & 10 & 12.16 & 9.78 \\
\hline 30 & Роааппиа & 8 & 18.37 & 19 & 18.98 & 15.85 \\
\hline 31 & Polygonumplebeium & 5.33 & 4.14 & 4.33 & 4.48 & 2.51 \\
\hline 32 & Prunella vulgaris & 6.67 & 5.33 & 4.67 & 8.10 & 7.11 \\
\hline 33 & Ranunculus arvense & 10.67 & 4.74 & 5 & 3.84 & 4.59 \\
\hline 34 & Rhumexhistatus & - & - & - & 7.47 & 6.07 \\
\hline 35 & Rumexnepalensis & - & 2.37 & 4 & 3.84 & 3.11 \\
\hline 36 & Salvia moorcraftiana & - & - & - & 7.89 & 6.37 \\
\hline 37 & Scandix pectin veneris & - & - & - & 5.12 & 3.70 \\
\hline 38 & Sonchusoleraceous & - & 4.14 & 12.33 & 11.09 & 9.48 \\
\hline 39 & Solanumnigrum & - & - & 6 & 4.27 & 5.78 \\
\hline 40 & Sorghum halepensis & - & - & - & - & 7.85 \\
\hline 41 & Stellaria media & - & 10.07 & 12 & 10.88 & 16.59 \\
\hline 42 & Taraxicumofficinale & - & - & 10.67 & 8.53 & 6.51 \\
\hline 43 & Thymus linearis & - & - & 11.33 & 12.8 & 10.51 \\
\hline 44 & Trifoliumpratense & - & 12.44 & 16.67 & 19.41 & 18.37 \\
\hline 45 & Trifoliumrepens & 8 & 15.40 & 22.33 & 26.45 & 29.92 \\
\hline 46 & VerbascumThapsus & - & - & 11 & 9.81 & 8 \\
\hline 47 & Veronica persica & - & - & - & 16.21 & 18.37 \\
\hline 48 & Xanthium spinosum & - & - & - & - & 3.11 \\
\hline & Total & 234.67 & 315.85 & 424 & 571.52 & 607.26 \\
\hline
\end{tabular}


Table.3 Influence of different sizes of quadrat on frequency (\%) of herbaceous species

\begin{tabular}{|c|c|c|c|c|c|c|}
\hline \multirow{2}{*}{$\begin{array}{c}\text { S. } \\
\text { No. }\end{array}$} & \multirow[t]{2}{*}{ Species } & \multicolumn{5}{|c|}{ Sizes of quadrat } \\
\hline & & $0.5 \mathrm{~m} \times 0.5 \mathrm{~m}$ & $0.75 \mathrm{~m} \times 0.75 \mathrm{~m}$ & $1 \mathrm{~m} \times 1 \mathrm{~m}$ & $1.25 \mathrm{~m} \times 1.25 \mathrm{~m}$ & $1.5 \mathrm{~m} \times 1.5 \mathrm{~m}$ \\
\hline 1 & Achilleamillefolium & - & - & 44.44 & 44.44 & 44.44 \\
\hline 2 & Amaranthusviridis & - & - & - & 44.44 & 66.67 \\
\hline 3 & Artemisia indica & - & - & - & 44.44 & 44.44 \\
\hline 4 & Avenafatua & - & 22.22 & 33.33 & 55.56 & 55.56 \\
\hline 5 & Brumusjaponicas & - & 22.22 & 55.56 & 55.56 & 55.56 \\
\hline 6 & Capsellaburapastoris & 22.22 & 33.33 & 44.44 & 55.56 & 55.56 \\
\hline 7 & Cirsiumarvense & - & - & - & 22.22 & 22.22 \\
\hline 8 & Centureaiberica & 22.22 & 44.44 & 44.44 & 55.56 & 55.56 \\
\hline 9 & Chenopodium album & - & - & 22.22 & 22.22 & 22.22 \\
\hline 10 & Convolvulus arvense & 33.33 & 33.33 & 44.44 & 55.56 & 55.56 \\
\hline 11 & Conyzacanadensis & 44.44 & 66.67 & 77.78 & 77.78 & 77.78 \\
\hline 12 & Chaerophyllumvillossum & - & - & - & - & 55.56 \\
\hline 13 & Cynodondactylon & 77.78 & 77.78 & 88.89 & 100 & 100 \\
\hline 14 & Erodiumcicutarium & - & - & - & 44.44 & 55.56 \\
\hline 15 & Euphorbia helioscopia & - & - & - & 44.44 & 44.44 \\
\hline 16 & Gallium aparine & 33.33 & 33.33 & 44.44 & 44.44 & 33.33 \\
\hline 17 & Geranium pratense & 11.11 & 22.22 & 44.44 & 44.44 & 33.33 \\
\hline 18 & Hypericumperforatum & - & - & - & 33.33 & 33.33 \\
\hline 19 & Lactucadissecta & 22.22 & 33.33 & 33.33 & 44.44 & 55.56 \\
\hline 20 & Lepidiumrudrale & 11.11 & 33.33 & 33.33 & 55.56 & 55.56 \\
\hline 21 & Lespedizacapitata & 11.11 & 33.33 & 33.33 & 44.44 & 44.44 \\
\hline 22 & Lolliumspp & - & - & - & 55.56 & 66.67 \\
\hline 23 & Marubiumvulgare & 11.11 & 33.33 & 44.44 & 44.44 & 55.56 \\
\hline 24 & Medicago minima & 11.11 & 33.33 & 44.44 & 66.66 & 66.67 \\
\hline 25 & Medicagopolymorpha & - & 22.22 & 22.22 & 22.22 & 22.22 \\
\hline 26 & Oenotherarosea & - & - & 44.44 & 44.44 & 55.56 \\
\hline 27 & Oxalis corniculata & 11.11 & 22.22 & 22.22 & 55.56 & 55.56 \\
\hline 28 & Plantagolanceolata & 11.11 & 44.44 & 44.44 & 66.67 & 66.67 \\
\hline 29 & Poabulbusa & 33.33 & 22.22 & 44.44 & 55.56 & 55.56 \\
\hline 30 & Pоаaпnиа & 44.44 & 66.67 & 66.67 & 66.67 & 66.67 \\
\hline 31 & Polygonumplebeium & 22.22 & 22.22 & 22.22 & 22.22 & 22.22 \\
\hline 32 & Prunella vulgaris & 22.22 & 22.22 & 22.22 & 55.56 & 55.56 \\
\hline 33 & Ranunculus arvense & 44.44 & 22.22 & 22.22 & 22.22 & 33.33 \\
\hline 34 & Rhumexhistatus & - & - & - & 44.44 & 44.44 \\
\hline 35 & Rumexnepalensis & - & 11.11 & 22.22 & 22.22 & 22.22 \\
\hline 36 & Salvia moorcraftiana & - & - & - & 44.44 & 44.44 \\
\hline 37 & Scandix pectin veneris & - & - & - & 33.33 & 33.33 \\
\hline 39 & Sonchusoleraceous & - & 22.22 & 55.56 & 66.67 & 66.67 \\
\hline 39 & Solanumnigrum & - & - & 33.33 & 22.22 & 44.44 \\
\hline 40 & Sorghum halepensis & - & - & - & - & 44.44 \\
\hline 41 & Stellaria media & - & 44.44 & 44.44 & 55.56 & 55.56 \\
\hline 42 & Taraxicumofficinale & - & - & 44.44 & 44.44 & 44.44 \\
\hline 43 & Thymus linearis & - & - & 55.56 & 55.56 & 55.56 \\
\hline 44 & Trifoliumpratense & - & 55.56 & 55.56 & 55.56 & 55.56 \\
\hline 45 & Trifoliumrepens & 22.22 & 55.56 & 55.56 & 66.67 & 77.78 \\
\hline 46 & Verbascumthapsus & - & - & 55.56 & 66.67 & 66.67 \\
\hline 47 & Veronica persica & - & - & - & 55.56 & 55.56 \\
\hline 48 & Xanthium spinosum & - & - & - & - & 33.33 \\
\hline & Average & 10.87 & 19.90 & 30.55 & 45.83 & 50.69 \\
\hline
\end{tabular}

$$
\text { C.D }(\mathrm{P} \leq 0.05)=5.36
$$


Table.4 Influence of different sizes of quadrat on abundance (plants $/ \mathrm{m}^{2}$ ) of herbaceous species

\begin{tabular}{|c|c|c|c|c|c|c|}
\hline \multirow{2}{*}{$\begin{array}{l}\text { S. } \\
\text { No. }\end{array}$} & \multirow[t]{2}{*}{ Species } & \multicolumn{5}{|c|}{ Sizes of quadrat } \\
\hline & & $\begin{array}{c}0.5 \mathrm{~m} \times \\
0.5 \mathrm{~m}\end{array}$ & $\begin{array}{c}0.75 \mathrm{~m} \times \\
0.75 \mathrm{~m}\end{array}$ & $1 \mathrm{~m} \times 1 \mathrm{~m}$ & $\begin{array}{c}1.25 \mathrm{~m} \times \\
1.25 \mathrm{~m}\end{array}$ & $1.5 \mathrm{~m} \times 1.5 \mathrm{~m}$ \\
\hline 1 & Achilleamillefolium & - & - & 6.5 & 7.36 & 7.56 \\
\hline 2 & Amaranthusviridis & - & - & - & 13.44 & 9.07 \\
\hline 3 & Artemisia indica & - & - & - & 5.12 & 5.56 \\
\hline 4 & Avenafatua & - & 5.33 & 9.5 & 18.56 & 15.33 \\
\hline 5 & Brumusjaponicas & - & 14.22 & 18 & 17.6 & 15.78 \\
\hline 6 & Capsella bursa pastoris & 12 & 17.78 & 19 & 16 & 12.67 \\
\hline 7 & Cirsiumarvense & - & - & - & 8.32 & 7.56 \\
\hline 8 & Centureaiberica & 16 & 21.33 & 14.5 & 14.08 & 12.89 \\
\hline 9 & Chenopodiumalbum & - & - & 8 & 6.4 & 6.67 \\
\hline 10 & Convolvulus arvense & 18 & 18.67 & 28 & 25.28 & 24.44 \\
\hline 11 & Conyzacanadensis & 28 & 24.89 & 11.67 & 26.67 & 26.96 \\
\hline 12 & Chaerophyllumvillossum & - & - & - & - & 7.78 \\
\hline 13 & Cynodondactylon & 172 & 165.92 & 145.83 & 164.90 & 206.34 \\
\hline 14 & Erodiumcicutarium & - & - & - & 10.24 & 6.59 \\
\hline 15 & Euphorbia helioscopia & - & - & - & 16.64 & 14 \\
\hline 16 & Gallium aparine & 20 & 9.78 & 10.5 & 11.52 & 14.22 \\
\hline 17 & Geraniumpretense & 20 & 14.22 & 8.5 & 3.84 & 13.33 \\
\hline 18 & Hypericumperforatum & - & - & - & 4.16 & 6.44 \\
\hline 19 & Lactucadissecta & 12 & 8.89 & 11 & 13.76 & 11.33 \\
\hline 20 & Lepidiumrudrale & 4 & 17.78 & 15 & 16.32 & 14.22 \\
\hline 21 & Lespedizacapitata & 4 & 16 & 12 & 16.32 & 12.22 \\
\hline 22 & Lolliumspp & - & - & - & 4.16 & 13.11 \\
\hline 23 & Marubiumvulgare & 8 & 9.78 & 14.5 & 16 & 12.74 \\
\hline 24 & Medicagominima & 8 & 28.44 & 25 & 26.88 & 22.89 \\
\hline 25 & Medicagopolymorpha & - & 10.67 & 10 & 14.08 & 15.11 \\
\hline 26 & Oenotherarosea & - & - & 11 & 9.6 & 7.70 \\
\hline 27 & Oxalis corniculata & 8 & 14.22 & 18 & 20.16 & 19.78 \\
\hline 28 & Plantagolanceolata & 8 & 11.56 & 17 & 16.64 & 12.89 \\
\hline 29 & Poabulbusa & 6 & 14.22 & 24.5 & 21.76 & 18.44 \\
\hline 30 & Роааппиа & 6 & 27.56 & 28.5 & 28.48 & 23.78 \\
\hline 31 & Polygonumplebeium & 16 & 12.44 & 13 & 8.32 & 7.56 \\
\hline 32 & Prunellavulgaris & 20 & 16 & 14 & 9.49 & 8.59 \\
\hline 33 & Ranunculus arvense & 26 & 14.22 & 15 & 5.76 & 9.33 \\
\hline 34 & Rhumexhistatus & - & - & - & 17.28 & 14 \\
\hline 35 & Rumexnepalensis & - & 3.56 & 12 & 11.52 & 9.33 \\
\hline 36 & Salvia moorcraftiana & - & - & - & 11.84 & 9.56 \\
\hline 37 & Scandix pectin veneris & - & - & - & 10.56 & 7.33 \\
\hline 38 & Sonchusoleraceous & & 6.22 & 22 & 16.64 & 14.22 \\
\hline 39 & Solanumnigrum & - & - & 6 & 6.4 & 8.67 \\
\hline 40 & Sorghumhalepensis & - & - & - & - & 18.44 \\
\hline 41 & Stellariamedia & - & 22.22 & 29 & 20.16 & 28.67 \\
\hline 42 & Taraxicumofficinale & - & - & 24 & 19.2 & 14 \\
\hline 43 & Thymus linearis & - & - & 19.5 & 15.89 & 12.67 \\
\hline 44 & Trifoliumpratense & - & 22.22 & 32.5 & 35.84 & 33.11 \\
\hline 45 & Trifoliumrepens & 24 & 27.56 & 39 & 39.68 & 39.03 \\
\hline 46 & Verbascumthapsus & - & - & 19 & 14.72 & 12 \\
\hline 47 & Veronica persica & - & - & - & 30.4 & 32.22 \\
\hline 48 & Xanthium spinosum & - & - & - & - & 6.67 \\
\hline & Total & 436 & 575.70 & 711.5 & 848 & 882.81 \\
\hline
\end{tabular}


Table.5 Influence of different quadrant sizes on density $\left(\mathrm{m}^{-2}\right)$, frequency $(\%)$ and abundance $\left(\mathrm{m}^{-2}\right)$ of herbaceous species

\begin{tabular}{|c|l|c|c|c|}
\hline $\begin{array}{c}\text { S. } \\
\text { No. }\end{array}$ & \multicolumn{1}{|c|}{ Treatments } & Density & Frequency & Abundance \\
\hline $\mathbf{1}$ & T1 $(0.5 \mathrm{~m} \times 0.5 \mathrm{~m})$ & 234.67 & 10.88 & 436 \\
\hline $\mathbf{2}$ & $\mathrm{T} 2(0.75 \mathrm{~m} \times 0.75 \mathrm{~m})$ & 315.85 & 19.90 & 575.70 \\
\hline $\mathbf{3}$ & $\mathrm{T} 3(1 \mathrm{~m} \times 1 \mathrm{~m})$ & 424 & 30.55 & 711.5 \\
\hline $\mathbf{4}$ & $\mathrm{T} 4(1.25 \mathrm{~m} \times 1.25 \mathrm{~m})$ & 571.52 & 45.83 & 848 \\
\hline $\mathbf{5}$ & T5 $(1.5 \mathrm{~m} \times 1.5 \mathrm{~m})$ & 607.26 & 50.7 & 882.81 \\
\hline & C.D $(\mathbf{P} \leq \mathbf{0 . 0 5})$ & $\mathbf{4 0}$ & $\mathbf{5 . 3 6}$ & $\mathbf{5 5 . 7 4}$ \\
\hline
\end{tabular}

The result are in confirmaty with the results of Cox, (1990); Barbour et al., (1987) who reported that a plot should be large enough to include significant number of individuals, but small enough so that plants can be separated, counted and measured without duplication or omission of individuals and Moseley et al.,(1989) who reported that larger quadrants were more efficient statistically. Moreover Barbour et al., (1987) revealed that increasing quadrant size does not significantly increase the number of species encountered. The minimal sample area can then be determined from the species/area curve where the slope is nearly horizontal.

Papanastasis (1977) also reported that shapes did not produce significantly different results, but larger quadrats were more efficient statistically. The size of the quadrat influences the probability of each species occurring within the quadrat. Small quadrats result in low frequencies for most species and many uncommon species will not be sampled except with large samples (Brown, 1954).

For a randomly distributed species, all plot sizes will result in equally precise estimates and for a clumped distributed species greater precision will result when plots somewhat larger than the mean clump size are used (Kenkel et al., 1989). Mirreh et al., (1995) recommended that quadrant of $4 \mathrm{~m}^{2}$ size is recommended for desert region vegetation studies. If several species with great variable plant sizes, distributions, and densities are to be measured, more than one quadrant size may be required (Smith et al., 1987).

\section{References}

Aberdeen, J.E.C. 1957. The effect of quadrat size, plant size, and plant distribution on frequency estimates in plant ecology. Aust. J. Bot. 6, 47-58.

Anderson, K.L. 1942. A Comparison of line transects and permanent quadrats in evaluating composition and density of pasture vegetation of the tall prairie grass type. J. Am. Soc. Agron. 34, 805-822.

Barbour, M. G., Burk, J. H. and Pitts, W.D. 1987 Terrestrial Plant Ecology. Chapter 9: Method of sampling the plant community. Menlo Park, CA: Benjamin/Cummings Publishing Co. pp. 56

Brown, D. 1954. Methods of surveying and measuring vegetation. Jarrold and Sons Ltd., Norwich. 223 pp.

Clapham, A. R. 1932. The form of the observational unit in quantitative ecology. Journal of Ecology. 20: 192-197.

Clements, F. E. 1916. The quadrat method in teaching ecology. Plant Succession, pp. 423-437.

Cox, G. 1990.Laboratory manual of general ecology 6th Ed. Dubuque, Iowa: William C. Brown.

Dombois, M. and Ellenberg, H. 1974. Aims and 
methods of vegetation ecology. John Wiley and Sons, Canada.

Gleason, H. A. 1920. Some applications of the quadrat method. Journal of Torrey Botonical Society, 47:21-33

Hines, W.T. 1984. Towards monitoring of longterm trends in terrestrial ecosystems. Environ

Kenkel, N. C., Juhasz-Nagy, P. and Podani, J. 1989. On sampling procedures in population and community ecology. Vegetation, 83: 195-207.

Krebs, C. J. 1999. Ecological methodology. Addison- Wesley Educational Publishers, Inc., Menlo Park, CA.

Menges, E.S., Gordon, D. R. 1996. Three levels of monitoring intensity for rare plant species. Nat

Mirreh, M. M., Al-Hassan, H.A and AlMonawer, F. S. 1995. Comparison of some vegetation inventory techniques in desert system. Ministry of Agriculture and Water Agricultural Research Management. Kingdom of Saudi Arabia.

Molnar, E.N., and Nosek, J.N. 1980. Spatial Processes in Grassland Community, II. Acta Bot. Acad. Sci. Hung. 26, 375-388.

Mosley, J.C., Bunting, S.C., and Hironaka, M. 1989. Quadrat and Sample sizes for frequency sampling mountain meadow vegetation. Great Basin Naturalist, 49: 241-248

Nosek, J.N. 1986. Spatial Processes in a Grassland Community, III. Acta Bot. Hung. 32, 61-78.

Papanastasia, V. P. 1977. Optimum size and shape of quadrat for sampling herbage weight in grasslands of northern Greece. Journal of Range Management,
30(6):446-448.

Philippi, T., Collins, B. and Guisti, S. 2001. A multistage approach to population monitoring for rare plant populations. Nat Areas J. 21:111-116

Pound, R. and Clement, F. E. 1900. Phytogeography of Nebraska, second edition, pp. 61-63.

Pound, R., and Clements, F. E. 1898.A method of determining the abundance of secondary species. Minnesota Botanical Studies, 2:19.

Sampson, A. W. 1914. Natural vegetation of range lands based upon growth requirements and life history of the vegetation. Journal on Agricultural Research, 3: 93-148.

Smith, S.D., Bunting, S.C., and Hironaka M., 1987.Evaluation of the improvement in sensitivity of nested frequency plots to vegetational change by Summation. Great Basin Naturalist, 47: 299-307.

Tahir, M. 2015. Plantation Techniques of Populus deltoids Bartr for Problematic Sites in Temperate Region of North Western Himalayas. Ph.D. Thesis submitted to Faculty of Forestry, SKUAST-K. 25pp.

Van Dyne, G.M., Vogel, W.G., and Fisser, H.G. 1987. Influence of Small Plot Size and Shape on Range Herbage Production Estimates. Ecology 44, 746-759 (1963). 10. Smith, S.D., Bunting, S.C., and Hironaka M., Evaluation of the Improvement in Sensitivity of Nested Frequency Plots to Vegetational Change by Summation. Great Basin Naturalist 47, 299-307.

\section{How to cite this article:}

Ishrat Saleem, J.A. Mughloo, A.H. Mughal and Afshan Anjum Baba. 2019. Quadrant Standardization for Herbaceous Species of Benhama, Ganderbal Area in Kashmir. Int.J.Curr.Microbiol.App.Sci. 8(03): 1697-1705. doi: https://doi.org/10.20546/ijcmas.2019.803.197 